\title{
EFFECT OF COHERENT IN-PLANE ANISOTROPY ON MAGNETIC ORDER IN AMORPHOUS HoFe ALLOYS
}

\author{
E. MASAGO, T. SAITO, K. SHINAGAWA and T.TSUSHIMA
}

Dept. of Physics, Faculty of Science, Toho Univ., Funabashi City, Chiba 274, Japan

\begin{abstract}
Effect of in-plane anisotropy of amorphous HoFe thin films on the magnetic phase transition was investigated by measurements of temperature dependence of magnetization. We found that the coherent in-plane anisotropy induces ferrimagnetic phase as perpendicular one does. Such a "coherent anisotropy" is important to stabilize ferrimagnetic phase of rare earth-transition metal films for magneto-optical storage.
\end{abstract}

\section{INTRODUCTION}

Recent studies have revealed that magnetic properties of amorphous rare earthtransition metal (RE-TM) alloys which are used as the magneto-optical recording media are not ordinary ferri(ferro)magnetic, but rather they should be treated as the random anisotropy magnets (RAM) [1], and the spin glass-like properties arise from frustration due to competition between the random local anisotropy field (D) and the ferromagnetic exchange field $(J)$ in alloys. For the magnetic system with small D/J (weak RAM), characteristic phases have been proposed by Chudnovsky et al. [2]; "correlated spin glass, (CSG)" and "ferri(ferro)magnet with wandering axes, FWA". In CSG state, the total magnetization (M) is zero, but ferromagnetic correlation is present. CSG state becomes FWA state with non zero $M$ under moderate external de filed $\mathrm{H}$, and therefore, the hysteresis curve of RAM looks like ferromagnetic one.

Chudnovsky et al. [2] proposed theoretically that the FWA phase is not also induced by $\mathrm{H}$ but also induced by coherent anisotropy as "perpendicular anisotropy" normal to the film plane. The RE-TM films prepared for magneto-optical storage have so strong perpendicular coherent anisotropy that FWA can be induced even without H. Recently, we showed for the first time experimentally [3] that the perpendicular anisotropy affects on the magnetic properties of amorphous (a-)DyGdFe thin films and a magnitude of perpendicular anisotropy brings ferromagnetic-like phase at a higher temperature side, though spin glass-like phase still appears at a lower temperature. In this paper, we extended the previous study [3], and investigated effect of "in-plane" anisotropy of a$\mathrm{HoFe}$ thin films on the magnetic order.

\section{EXPERIMENTS}

Amorphous $\mathrm{Ho}_{15} \mathrm{Fe}_{84}$ films with several magnitude of in-plane anisotropy, but with almost the same composition, were prepared by the conventional if sputtering technique 
onto glass substrates. The amorphous structures were checked by using $\mathrm{X}$-rays. We selected three samples with in-plane coherent anisotropy field of $8 \mathrm{kOe}$ (sample a), $9 \mathrm{kOe}$ (sample b), and $13 \mathrm{kOe}$ (sample $\mathrm{c}$ ) by measuring the magnetic hysteresis curves. The temperature dependence of both field-cooled (FC) and zero-field-cooled (ZFC) magnetizations was measured by a vibrating-sample-magnetometer. The dc field $\mathrm{H}$ was applied parallel to the film plane. There is no additional anisotropy when $\mathrm{H}$ is rotated in the film plane.

\section{RESULTS AND DISCUSSION}

Fig.1 shows temperature dependence of magnetizations divided by dc fields $(\mathrm{M} / \mathrm{H})$ for three a- $\mathrm{Ho}_{16} \mathrm{Fe}_{84}$ samples (a), (b) and (c) with different in-plane coherent anisotropy. $\mathrm{H}$ was fixed at $50 \mathrm{Oe}$. In this figure, we notice that 1) all samples exhibit thermal hysteresis; FC magnetization deviates from ZFC one at a temperature ( $\equiv T_{f}$ ) but 2 ), as magnitude of the coherent anisotropy becomes larger (from (a) to (c)), the irreversibility defined by the area between FC and ZFC magnetizations below $T_{f}$ becomes smaller; 3 ) the inflection point of magnetization (indicated as $T_{C}$ in Fig.1) shifts toward higher temperature side with increasing the in-plane anisotropy; and 4) the magnitude of $\mathrm{M} / \mathrm{H}$ becomes larger with increasing the inplane anisotropy. The compositions of the present samples are not yet analyzed, but we think that they have almost the same composition within 0.4 at \% taking ICP analysis for the other samples [3] into consideration. The above results suggest that as the in-plane coherent anisotropy becomes larger, the ferrimagnetic-like (FWA) long-range order is induced above $T_{f}$ and stabilized as discussed in our previous paper for perpendicular anisotropy [3]. We also point out that as

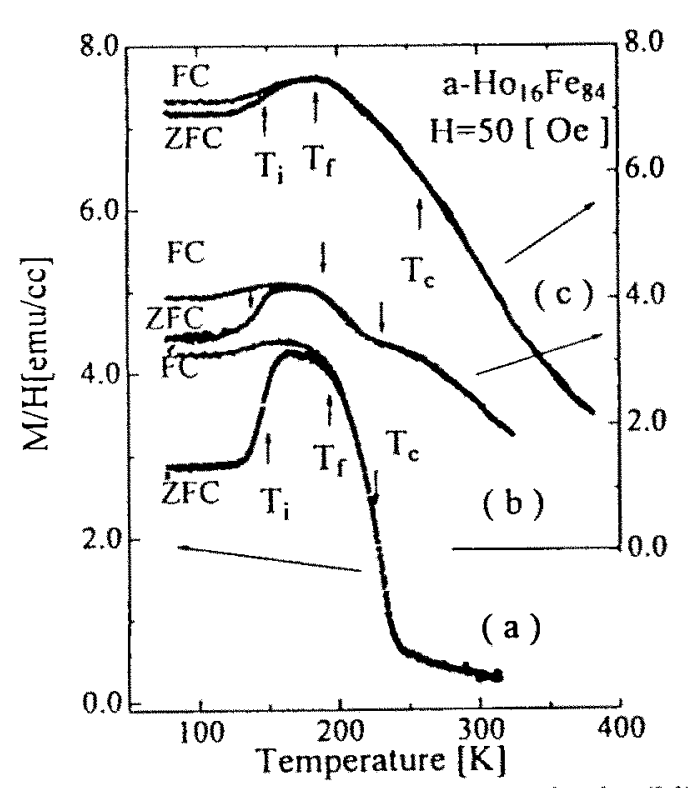

Fig.1 Temperture dependence of magnetization( $M$ ) divided by dc field(H) for a- $\mathrm{Ho}_{16} \mathrm{Fe}_{24}$ with different cohemt in-plane anisotropy fields $(\mathrm{CA}) ; \mathrm{CA}=8 \mathrm{kOe}$ (sample (a)), $\mathrm{CA}=9 \mathrm{kOe}($ sample(b)), and $\mathrm{CA}=13 \mathrm{kOe}$ (sample(c)) shown in Fig.1, magnetization of sample (b) has a shoulder at $T_{C}$, and intermediate behavior between that of sample (a) and (c) is observed. It implies that the in-plane anisotropy field of about $9 \mathrm{kOe}$ is critical to induce long-range ferrimagnetic-like order, that is, below $9 \mathrm{kOe}$ spin glass-like phase (CSG phase) is dominant when $\mathrm{H}$ is small.

We also measured the temperature dependence of magnetization as a function of $\mathrm{H}$. 
Such a measurement gives an important information in random magnets as RAM or spin glasses [1], because they have characteristic critical lines in H-T plane. Fig. 2 shows the $\mathrm{H}-\mathrm{T}$ phase diagrams of two a- $\mathrm{Ho}_{16} \mathrm{Fe}_{84}$ samples (a) and (c). For sample with in-plane coherent anisotropy field of $8 \mathrm{kOe}$ (Fig. 2(left)), the characteristic lines $H\left(T_{c}\right), H\left(T_{f}\right)$ and $H\left(T_{i}\right)$ in $H-T$ plane are similar to those of a-DyFe with small $D / J$ and with almost no coherent anisotropy, which is a standard weak RAM [1][3]. With decreasing temperature, in $\mathrm{H}=0$, the spin system freezes into randomly frozen CSG state. In the presence of $\mathrm{H}$, ferrimagnetic FWA phase is induced between $T_{c}$ and $T_{f}$, and in addition, the transverse and longitudinal components of spins to $H$ freeze independently at $T_{f}$ and $T_{i}$ like Heisenberg spin glasses, which is confirmed by the ac longitudinal and transverse susceptibilities in $H$ [1]. In the present work, $H\left(T_{i}\right)$ line was determined by the inflection temperature of $\mathrm{M}$ (see Fig.1).

For sample with in-plane coherent anisotropy of $13 \mathrm{kOe}$ (Fig. 2(right)), we notice that the $H-T$ phase diagram changes considerably. In region between $H\left(T_{c}\right)$ and $H\left(T_{f}\right)$, the FWA phase is considered to be induced even without $H$. (The data for $H=0$ was determined by ac susceptibility.) In addition, the $T_{c}$ becomes higher. The same tendency is shown in a-DyGdFe system with coherent "perpendicular" anisotropy [3]. For the present case of a-HoFe with in-plane anisotropy, Fig.1 and Fig.2 indicate that the FWA phase is induced and stabilized by the coherent "in-plane" anisotropy as well as by the perpendicular anisotropy.

In Fig.3, we compared the effect of "in-plane" coherent anisotropy in a-HoFe and that of "perpendicular" one in a-DyGdFe[3] on the characteristic temperatures. Fig.3
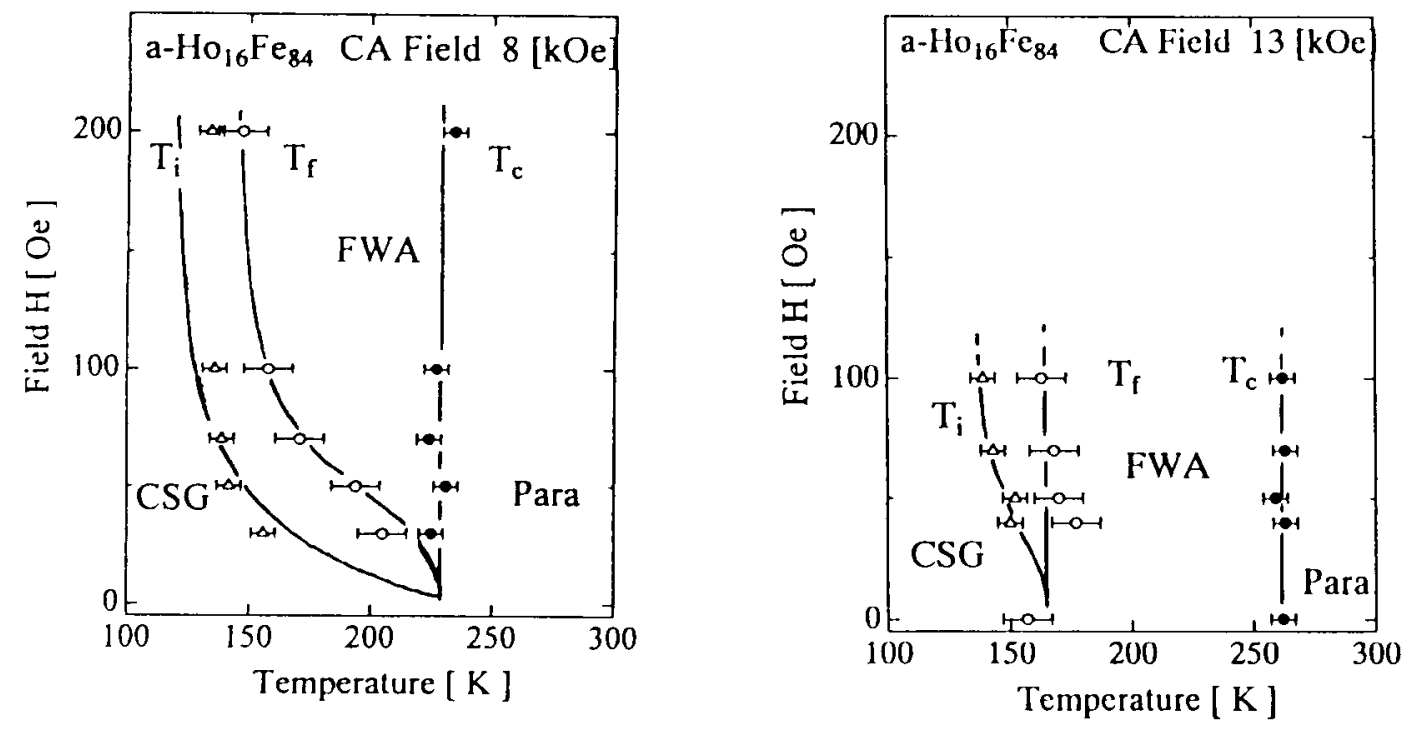

Fig.2 Characteristic lines in $\mathrm{H}$-T plane determined by dc magnetization measurements for sample with in-plane Coherent anisotropy $(\mathrm{CA})=8 \mathrm{kOe}$ (left), and sample with in-plane $\mathrm{CA}=13 \mathrm{kOe}$ (right) 
suggests that there is a critical coherent field that induces FWA phase without $\mathrm{H}$, and it is about $9 \mathrm{kOe}$ for the in-plane anisotropy field, and about $30 \mathrm{kOe}$ for the perpendicular anisotropy. The existence of such a critical anisotropy field has been theoretically proposed by Chudnovsky et al.[2], and by Y.Y.Goldschmidt et al. [4].

We note, however, that the data for a-HoFe was measured in the presence of external field of $50 \mathrm{Oe}$, though $\mathrm{H}=0$ for a-DyGdFe. Therefore the field effect, that FWA region is enlarged by $\mathrm{H}$, is included for a-HoFe. (Now, ac susceptibility measurement in $\mathrm{H}=0$ for a-HoFe is in progress.) However, the difference is very distinct even if the field effect is considered; the in-plane coherent anisotropy is more effective to induce FWA than perpendicular anisotropy. It is not clear at present why the in-plane anisotropy is so effective, though we cannot exclude the fact that $\mathrm{D} / \mathrm{J}$ of a-HoFe is larger than that of aDyGdFe [5]. To check the effect of the

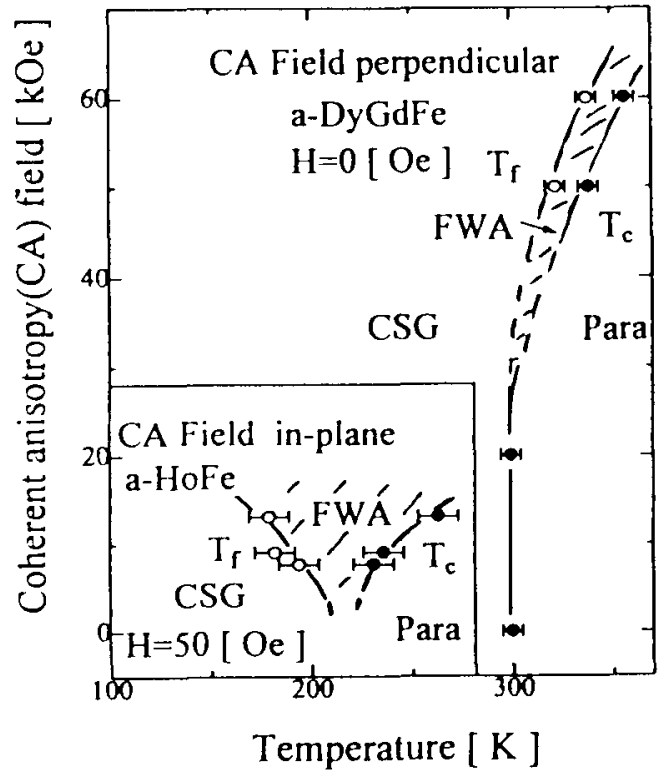

Fig.3 Effect of coherent anisotropy(CA) field on magnetic phases of a-DyGdFe with perpendicular anisotropy[3] and a-HoFe with in-plane anisotropy. We note that $\mathrm{H}$ is not zero for a-HoFe. difference of $\mathrm{D} / \mathrm{J}$, the similar investigation on a-ErFe is also now in progress.

In conclusion, our results indicate that the (both in-plane and perpendicular) coherent anisotropy is very important factor to determine the magnetic phase transitions of amorphous RE-TM alloys, which are used as materials for magneto-optical storage.

\section{REFERENCES}

[1] T.Saito, Y.Matsumaru, K.Shinagawa and T.Tsushima, J.Magn.Magn.Mat. 130 (1994) 347; and references therein.

[2] E.M.Chudnovsky, W.M.Saslow and R.A.Serota, Phys.Rev.B33 (1986) 251.

[3] T.Saito, H.Miyano, K.Shinagawa and T.Tsushima, J.Magn.Magn.Mat. 140-144 (1995) 1757.

[4] Y.Y.Goldschmidt and A.Aharony, Phys. Rev. B32 (1985) 264.

[5] K.Imai, E.Masago, T.Saito, K.Shinagawa and T.Tsushima, J.Magn.Magn.Mat. (ICM'97) to be published. 